Puede deducirse el concepto de mol cuando el alumno conoce las masas de las partículas subatonicas. Conociendo el número atómico y el número másico puede calcular la masa atómica absoluta a partir de estos datos:

$$
{ }_{1 \mathrm{Na}}^{23}
$$

Puede deducirse fácilmente el número de protones y neutrones que posee el átomo de sodio. Estableciendo la relación entre la unidad de masa atómica y su equivalente en gramos:

$$
1 \text { uma }=1.66 \times 10^{-24} \mathrm{~g}
$$

Si bien la equivalencia puede darse con más precisión, en cursos de bachiflerato de los primeros años no es necesario una aproximación mayor, aunque desde Iuego esto no es objetable y queda a criterio del profesor utilizar nayor exactitud en la equivalencia.

Calculando el número de protones y neutrones se llegará a que el átomo de sodio tiene una masa absoluta de 23 umas. Luego:
1 uma $1.66 \times 10^{-24} \mathrm{~g}$ 23 uma $3.818 \times 10^{-25} \mathrm{~g}$.

Puede sugerirse a los alumnos que averigüen el número de átomos de sodio presentes en $23 \mathrm{~g}$ de sodio, lo que llevará a la siguiente conclusión:

$3,818 \times 10^{-25} \mathrm{~g} .-1$ átomo de $\mathrm{Na}$ $23 \mathrm{~g}$. - - $-6,02 \times 10^{23}$ átomos de $\mathrm{Na}$

Repitiendo este procedimiento con algunos ejemplos más, los alumnos llegan fácilmente a la definición de mol como "cantidad de materia que contiene $6,02 \times 10^{23}$ partículas", en este caso, átomos (1).

Cuando se conozca ya la formación de compuestos y tos alumnos tengan la noción de molécula, se aplicará el procedimiento para llegar al mol de moléculas y asi el alumno estará capacitado para relacionar en cada caso la ecuación química con la correspondiente relación estequiométrica, por ejemplo:

$1 \mathrm{~mol}$ de moléculas de oxigeno reacciona con 2 moles de moléculas de hidrógeno para dar 2 moles de moléculas de agua $\left(\mathrm{O}_{2}+2 \mathrm{H}_{2} \div 2 \mathrm{H}_{2} \mathrm{O}\right)$.
De la misma manera cuando escriba las ecuaciones correspondientes a la formación de óxidos, hidróxidos, ációos y sales. De cste modo, relacionando siempre las ecuaciones químicas con sus correspondientes relaciones estequiométricas, se evita la disociación que ocurre habitualmente, y en cada caso es fácil hacer pequeños problemas re. lacionando masas y moles, etc.

Nota:

(1) Diversos autores comparten este criterio; asi se presenta en:

Staff or Research and Education Association, 1986, The Chemistry problem solver, (Research and Education Association: New York).

Bella, Alicia, 1984, Comunicación a la REQ JI. San Juan, 1984.

Beltrán, Faustino, 1980, Fórmulas químicas ranozadas. (Plus Ultra. Buenos Aires).

Nella Bonetto de Fushimi Liceo Victor Mercante. UNLP. La Plata. Argentina.

\title{
EXPERIENCIAS DE CLASE
}

\section{ESTUDIO CINÉTICO DE UNA REACCIÓN DE COMBUSTIÓN}

La cinética química es uno de los temas presentes en el programa de la Química de COU y en el plan de estudios de Escuelas Universitarias de formación del Profesorado de EGB.

Nuestra experiencia docente nos ha hecho constatar, la dificultad que encuentran los alumnos a la hora de adquirir y asimilar cada uno de los conceptos básicos relacionados con el tema.

Dada la conexión que existe entre la adquisición de conocimientos y las experiencias desarrolladas por los alumnos, creemos oportuno introducir la cinética química mediante una metodologia activa, a través de la cual, pondrán en práctica el método científico.

Para desarrollar los conceptos básicos implicados en el tema, tales como: relación concentración-tiempo, velocidad y orden de reacción, catálisis, etc; y con objeto de poner al estudiante en con- tacto con la construcción de gráficas e interpretación de las mismas, proponcmos el estudio de una reaccion de combustión.

Para esta reacción utilizamos comburentes adecuados, lo que permite controlar la velocidad de la reacción, haciéndola suficientemente lenta para que Ios alumnos puedan medir tiempos con facilidad con ayuda de un cronómetro.

Como comburente se utiliza nitrato de potasio y como combustible mechas de algodón del tipo $6 \mathrm{c}$.

Cada gnupo de alumnos preparará 6 disoluciones de nitrato de potasio cuyas concentraciones varían desde $0.25 \mathrm{M}$ hasta disolución saturada, a la temperatura ambiente. A continuación sumergirán en cada disolución una mecha de hifo de algodón de $30 \mathrm{~cm}$. de longitud. El algodón se empapará de disolución, de modo que la cantidad de sustancia absorbida por unidad de longitud será proporcional a la concentración de la disolución preparada.
Después se secará completamente el hilo de algodón con un secador de mano. Esta condición es imprescindible, ya que si el hilo está humedo, no habrá una combustión adecuada.

Se iniciará la combustión ascendentes de cada hilo de algodón acercando un ascua y se medirá con el cronómetro el tiempo que tarda en quemarse cada mecha.

Para la realización del estudio cinético los alumnos representarán las concentraciones (eje $X$ ) frente a los tiempos de combustión (eje Y) de las diferentes muestras. Se aprecia en dicha gráfica como a medida que aumenta la concentración de nitrato en el hilo, disminu. ye el tiempo de combustión ajustándose la curva a una exponencial.

$\mathrm{Si}$ ahora se calculan los logaritmos de las concentraciones y se representan (eje Y), frente a los tiempos (eje X), se obtiene una linea recta de pendiente negativa, concluyéndose que la cinetica es de primer orden, respecto a la concentración. 
En una segunda parte de la experiencia los alumnos comprueban como las mezclas nitrato-clorato y nitratoperclorato, aumentan la velocidad de combustión, pudiendo considerar al clorato y perclorato sustancias que actuan como catalizadores positivos. Por el contrario, las mezclas nitratobicarbonato y nitrato-sulfato de hierro (II), disminuyen la velocidad de la reacción, considerando al bicarbonato y sulfato de hierro como catalizadores negativos.

Con este experimento nuestros alumnos estudjan uno de los factores más importantes que interviene en la cinética quimica, a saber, la variación de la velocidad de una reacción con la concentración y con la adición de sustancias que actúan como catalizadores.

La manera en la cual la velocidad de una reacción depende de la concentracion de los reaccionantes se expresa cuantitativamente por la ecuación de velocidad obtenida.

Dada la dificultad que presenta la cinética química a la hora de hacer el seguimiento de las sustancias reaccionantes o de los productos formados, a través de técnicas de análisis complejas, pensamos que esta experiencia, realizada durante una sesión de laboratorio, aporta una metodología interesante, a través de la cual los alumnos pueden asimitar mejor los conceptos aquí mencionados.

Isabel Carrascal Nieto, M. Carmen García Gómez. Escuela Universitaria de Formación del Profesorado de EGB Santa Maria. Madrid.

Pedro Gonzalo Romera, I.B. Gran Capitán, Madrid.

\section{LA FORMULACIÓN EN EL LABO-} RATORIO. UN VÍDEO DIDÁCTICO.

\section{Introducción}

Dada la dificultad de asimilación de numerosos conceptos abstractos en el proceso de aprendizaje de las ciencias, co- mo son la formulación y nomenclatura quimicas, hemos venido desarrollando durante los últimos cursos distintas metodologías dirigidas a facilitar la enseñanza-aprendizaje de este tema en concreto (Martinez Aznar y Ovejero Morcillo, 1986).

Por ello, surgió la idea de realizar un video que permitiera relacionar el lenguaje químico abstracto y los hechos experimentales por él representados.

\section{Desarrollo}

El video consta de dos partes:

- En la primera parte se establece la división de las sustancias quimicas puras, incidiendo en la diferencia existente entre elementos y compuestos. Se destaca la necesidad de la nomenclatura quimica, nos referimos a la procedencia del nombre y simbolo de los elementos a través de ejemplos $y$, por último, se hace alusión a las fórmulas quimicas, expresiones que indican los elementos presentes en un compuesto, así como la proporción en que intervienen.

- La segunda parte consta de cinco experiencias de laboratorio que permiten visualizar la obtención de compuestos binarios, agrupados según cl siguiente esquema Tabla 1.

En todos los casos, las experiencias ele gidas, aunque no constituyen el método más frecuente para la obtención de los productos (las hemos realizado a partir de los elementos), presentan las ventajas de ser fáciles de ententer y reproducir por los alumnos.

Cono punto de partida de esta segunda parte se hace referencia a la oxidación como ejemplo de reacción química corriente en la vida cotidiana. Este hecho nos permite introducir las reacciones del oxigeno con diversos elementos en dos experiencias (Tabla 2).

Después de ver las reacciones con oxígeno examinaremos las de otro elemento que ongina gran número de compuestos, el hidrógeno. En este vídeo no se realiza la obtención de hidruros metálicos dada la peligrosidad que supondria para el alumno el manejo de los productos requeridos (Tabla 3).

Por último se realizan experiencias de combinaciones de los restantes elementos entre si (Tabla 4).

El vido finaliza con una recapitulación de los compuestos obtenidos, denominados binarios por presentar dos tipos de átomos, incluyéndose su clasificación, que vimos al principio de este trabajo.

\section{Conclusiones}

Aunque la orientación inicial sea la formulación y nomenclatura quimicas, este video permite la introducción de otros aspectos de la química, como reactividad (factores cinéticos y termodinámicos), propiedades y manejo de las sustancias, tipos de reacciones y en especial reacciones redox, etc. La variedad de conceptos reseñados permite su aplicación a distintos niveles educativos, en un variado grado de profundización. Por último, el vídeo apoya la necesidad de la experimentación en todo proceso de enseñanza-aprendizaje de las Ciencias.

\section{Bibliografia}

Martínez Aznar, M* M. y Ovejero Morcillo, P., 1986, La Enseñanzaaprendizaje de la Formulación y Nomenclatura Química (Una aproximación al Método Keller), $X X I$ Reunión Bienal de la R.S.E.Q., pp. 152.

Martínez Aznar, $\mathrm{M}^{\mathrm{a}}$ Mercedes, ' Ovcjero Morcillo, Paloma, ${ }^{2}$ Bergondo Llorente, Eladio, ${ }^{3}$ Gutiérrez. Alonso, Angel."

(1) Departamento de Didáctica de las Ciencias Experimentales.

E.U. "Maria Diaz Jiménez», Universidad Complutense

Avda. Islas Filipinas, 3, Madrid. (2) I.N.B. «Jorge Guillèn» Alcorcón, Madrid.

(3) C.E.P. Alcorcón, Madrid.

(4) Dpto. Quimica Inorgánica, Universidad Complutense, Madrid. 


\section{ESTUDIO CUANTITATIVO DEL EQUILIBRIO QUIMICO POR VIA EXPERIMENTAL}

Es evidente que en el mundo en el cual nos movemos existe una serie de fenómenos que merecen llamar nuestra atención, pero yo tengo que reconocer mi debilidad hacia el equilibrio quimico, encontrando fascinante el cómo se puede desplazar la reacción en uno u otro sentido al variar la concentración de uno de los componentes, con objeto de mantener invariable su constante de equilibrio.

El estudio se realiza desde los aspectos cualitativos y cuantitativos, buscando reacciones de fácil montaje. Para los aspectos cualitativos se elige el equilibrio: $\mathrm{Cro}_{4}^{2-}$ (aq.) $\simeq \mathrm{CrO}_{7}^{2-}$ (aq.).

Desde el punto de vista cuantitativo, nos hemos sentido vivamente interesados por la comprobación de la constante de equilibrio de la ley de accion de masas. El buscar la reacción adecuada conforme a los recursos de nuestro Laboratorio, trajo consigo una revisión de bibliografia, observando la escasez de la misma.

El cquilibrio propuesto para su estudio ha sido el siguiente:

$\mathrm{H}_{2} \mathrm{C}_{2} \mathrm{O}_{4}+\mathrm{CaCl}_{2}=\mathrm{CaC}_{2} \mathrm{O}_{4}+2 \mathrm{HCl}$

Comprobación de la constante de equilibrio de la ley de acción de masas

Aparatos: Balanza; bureta; pipeta; matraz aforado de 250 y de $1000 \mathrm{ml}$; embudo; vasos de precipitados de 50 y de $400 \mathrm{ml}$; papel de filtro.

Reactivos: Disoluciones valoradas de: Acido oxálico 0,25 M; Cloruro de calcio 0,25 M; Permanganato potásico 0, 1 $\mathrm{N}$; Acido clorhidrico 0,1 M; Hidróxido de sodio 0,1 M; Acido sulfúrico diluído $(1: 3)$.
Procedimientos:

1a) En un vaso de precipitados de 50 $\mathrm{ml}$, se mezclan $10 \mathrm{ml}$ de disolución 0,25 $M$ de ácido oxálico con otros $10 \mathrm{ml}$ de disolución $0,25 \mathrm{M}$ de cloruro cálcico. Se produce un precipitado: $\mathrm{H}_{2} \mathrm{C}_{2} \mathrm{O}_{4}+$ $\mathrm{CaCl}_{2} \rightarrow \mathrm{CaC}_{2} \mathrm{O}_{4}+2 \mathrm{HCl}^{2}$

Si la reacción fuese completa desaparecerian todo el oxálico, pero no es asi como se comprueba al filtrar el líquido sobre un filtro seco, recogiendo el filtrado en un vaso también seco, repitiendo la operación sobre el mismo fi]tro hasta conseguir un líquido completamente claro.

Se valoran 10 mi de este liquido con la disolución $0,1 \mathrm{~N}$ de permanganato potásico. Con estos datos se calcula ia relación entre las concentraciones del áci. do clorhídrico, del ácido oxálic̣o y del cloruro de calçio libre.

1b) Repetir lo descrito en Ia), pero agregando antes de los $10 \mathrm{ml}$ del $\mathrm{CaCl}_{2}, 10 \mathrm{ml}$ de ácido clorhídrico. Calcular finalmente las nuevas concentraciones de equilibrio.

1c) Repetir lo descrito en 1b), pero sustituyendo los $10 \mathrm{ml}$ de $\mathrm{HCl}$ por $10 \mathrm{ml}$ de disolución de hidróxido de sodio 0,1 M. Calcular finalmente las nuevas concentraciones de equilibrio.

\section{Resumen: Primera Experiencia}

Temperatura del laboratorio $13^{\circ} \mathrm{C}$; Disolución de ácido oxálico $0,2486 \mathrm{M}$; Disolución de cloruro de calcio $0,25 \mathrm{M}$; Temperatura de valoración $75^{\circ} \mathrm{C}$.

Datos: $1 \mathrm{ml}$ de $\mathrm{KMnO}_{4}$ se equivale con $4,499 \cdot 10^{-3} \mathrm{~g}$ de ácido oxálico. $1 \mathrm{~g}$ de ácido oxálico se equivale con $0,81 \mathrm{~g}$ de $\mathrm{HCl}$.

En cualquiera de las experiencias llevadas a cabo, se observa perfectamente el desplazamiento del equilibrio; en la adición de ácido $\mathrm{HCl}$ el equilibrio se en- cuentra desplazado hacia ta izquierda con mayor formación de $\mathrm{C}_{2} \mathrm{O}_{4} \mathrm{H}_{2}$ y más cantidad de $\mathrm{CaCl}_{2}$; en la adición de $\mathrm{NaOH}$ cl equilibrio se desplaza hacia la derecha y por tanto menor cantidad de ácido oxálico libre y de cloruro de calcio.

\section{Consideraciones finales}

Con objeto de cometer menos errores es preferible no valorar $10 \mathrm{mi}$ de la disolución del equilibrio, sino todo el volumen que se ha obtenido de! filtrado.

Se observa que los valores más discordantes los da siempre el equilibrio con adición de $\mathrm{HCl}$.

Los mejores resultados se obtienen efectuando las valoraciones a temperaturas comprendidas entre $70-80^{\circ} \mathrm{C}$, siendo esta última la que ha dado mayor concordancia.

La experiencia cuarta en que sc dejó en reposo ci sistema durante 24 horas antes de proceder a su valoración, es la que ha dado los valores más discordantes, por tanto en esta reacción no interesa dicho reposo.

Los valores oblenidos para la constante de equilibrio dentro de cada experiencia son admisibles, de todas formas las conclusiones serian más fidedignas si se hubiera operado con productos más puros, para lo cual habria que haber recurrido a la casa Merck y a un cloruro de calcio anhidro del $95 \%$ de pureza.

Los cálculos operacionales, están a disposición de quien le pueda interesar.

María G. Souto Figueroa, Catedrática de Física y Química del I.B. ce Carbaltiño (Orense) 